\title{
A photometric study of the W UMa-type system U Pegasi
}

\author{
G. Djurašević ${ }^{1}$, H. Rovithis-Livaniou ${ }^{2}$, P. Rovithis ${ }^{3}$, S. Erkapić $^{1}$, and N. Milovanović ${ }^{1}$ \\ 1 Astronomical Observatory, Volgina 7, 11050 Belgrade, Yugoslavia \\ 2 Section of Astrophysics-Astronomy \& Mechanics, Dept. of Physics, Athens University, GR Zografos 15784 , \\ Athens, Greece \\ e-mail: elivan@atlas.uoa.gr \\ 3 Astron. Institute, National Observatory of Athens, PO Box 20048, 11810 Athens, Greece
}

Received 14 April 2000 / Accepted 20 November 2000

\begin{abstract}
In the present study, the activity of the eclipsing binary of the W UMa-type system U Peg is examined by analysing the photoelectric observations covering the period from 1950 to 1989. During this period, the light curves show significant differences and asymmetries. The analysis of the corresponding light curves is made using Djurašević's inverse problem method. To explain the light-curve asymmetries and variations, we used a Roche model that involved regions containing spots on the components. The analysis shows that the system U Peg is in an overcontact configuration $\left(f_{\text {over }} \sim 14.9 \%\right)$. The Roche model with spotted areas on the cooler component yields a good fit of the observations for the whole set of the analysed light curves without any changes of the basic system parameters. This indicates that the complex nature of the light-curve variations during the examined period can be explained by the evolution and motion of spotted areas on the cooler component. According to the obtained results, the spotted areas cover a significant part of the stellar surface; the changes in their location and size with time are examined.
\end{abstract}

Key words. stars: individual: U Peg - binaries: close: starspots - stars: activity

\section{Introduction}

The variability of $\mathrm{U} P$ eg $\left(\mathrm{BD}+15^{\circ} 4915\right)$ was discovered by Chandler (1895) and the system belongs to the W subclass of the W UMa-type eclipsing binaries. From photoelectric observations it was found that its light curves are variable.

Regarding the spectral type of U Peg, Adams et al. (1924) classified it as F3, but later this was revised to G3 (Adams et al. 1935). Struve et al. (1950) estimated the radial velocities of the two components from 4 spectra of the system, but the most recent and most accurate radial velocity observations were made by $\mathrm{Lu}$ (1985).

Some of the light curves of the system have been analysed previously by different methods; e.g. using the classical Russell model (Binnendijk 1960), Kopal's frequency domain techniques (Lafta et al. 1986) or W-D code (Russo et al. 1982; Zhai et al. 1984; Zhai et al. 1988). In the present paper, photoelectric observations (La Fara 1952; Binnendijk 1960; Rigterink 1972; Zhai et al. 1984 and

Send offprint requests to: G. Djurašević, e-mail: gdjurasevic@aob.bg.ac.yu
Maupomé et al. 1991) of U Peg were analysed to study the activity of the system and to estimate the orbital and physical parameters of U Peg.

\section{Light-curve analysis}

To analyse these asymmetric light curves, probably deformed by the presence of spotted areas on the components, we used Djurašević's (1992a) programme generalised to the case of an overcontact configuration (Djurašević et al. 1998). The programme is based on the Roche model and the principles arising from the paper by Wilson \& Devinney (1971). The light-curve analysis was made by applying the inverse-problem method (Djurašević 1992b) based on Marquardt's (1963) algorithm.

According to this method, the stellar size in the model is described by the filling factors for the critical Roche lobes $F_{1,2}$ of the primary and secondary component, respectively, which tell us to what degree the stars in the system fill their corresponding critical lobes. For synchronous rotation of the components these factors are expressed as the ratio of the stellar polar radii, $R_{1,2}$, and the corresponding polar radii of the critical Roche lobes, i.e., 
$F_{1,2}=R_{1,2} / R_{\text {Roche }_{1,2}}$. In the case of an overcontact configuration the potential $\Omega_{1,2}$ characterising the common photosphere is derived with the filling factor of the critical Roche lobe $F_{1}>1$ of the primary, while the factor $F_{2}$ may be excluded from further consideration. The degree of overcontact is defined in the classical way (Lucy \& Wilson 1979) as:

$f_{\text {over }}[\%]=100 \cdot\left(\Omega_{1,2}-\Omega_{\mathrm{i}}\right) /\left(\Omega_{\mathrm{o}}-\Omega_{\mathrm{i}}\right)$,

where $\Omega_{1,2}, \Omega_{\mathrm{i}}$, and $\Omega_{\mathrm{o}}$ are the potentials of the common photosphere and of the inner and outer contact surfaces, respectively. To achieve more reliable estimates of the model parameters in the light-curve analysis programme, we applied a quite dense coordinate grid, having $72 \times 144=10368$ elementary cells per star. The intensity and angular distribution of radiation of elementary cells are determined by the stellar effective temperature, limb-darkening, gravity-darkening and by the effect of reflection in the system.

The presence of spotted areas (dark or bright) enables us to explain the asymmetries and the light-curve anomalies. In our code these active regions are approximated by circular spots, characterised by the temperature contrast of the spot with respect to the surrounding photosphere $\left(A_{\mathrm{S}}=T_{\mathrm{S}} / T_{*}\right)$, by the angular dimension (radius) of the spot $\left(\theta_{\mathrm{S}}\right)$ and by the longitude $\left(\lambda_{\mathrm{S}}\right)$ and latitude $\left(\varphi_{\mathrm{S}}\right)$ of the spot centre. The longitude $\left(\lambda_{\mathrm{S}}\right)$ is measured clockwise (as viewed from the direction of the $+Z$-axis) from the $+X$-axis (line connecting star centres) in the range $0^{\circ}-360^{\circ}$. The latitude $\left(\varphi_{\mathrm{S}}\right)$ is measured from $0^{\circ}$ at the stellar equator (orbital plane) to $+90^{\circ}$ towards the "north" $(+Z)$ and $-90^{\circ}$ towards the "south" $(-Z)$ pole.

For a successful application of this model in the analysis of the observed light curves, the method proposed by Djurašević (1992b) was used. Optimum model parameters are obtained through the minimization of $S=\Sigma(\mathrm{O}-\mathrm{C})^{2}$, where $\mathrm{O}-\mathrm{C}$ is the residual between the observed (LCO) and synthetic (LCC) light curves for a given orbital phase. The minimization of $S$ is done in an iterative cycle of corrections of the model parameters. In this way the inverseproblem method gives us the estimates of system parameters and their standard errors.

In the analysis of the light curves, instead of the often used and somewhat questionable practice of forming normal points, we used the original observational data in order to avoid negative influences of such normalization. La Fara's (1952) observations are an exception because only the normal points published by the author were available to us. The present light-curve analysis shows that during the deeper (primary) minimum the cooler (more-massive and larger) component eclipses the hotter (less-massive and smaller) one. Since the primary minimum is an occultation, the mass-ratio of the components was fixed in the inverse problem at Zhai et al.'s (1988) simultaneous (photometric and radial velocity) solution $q=m_{\mathrm{c}} / m_{\mathrm{h}} \sim 3.019$. Based on the spectral type (G2 V; Zhai et al. 1988) the temperature of the less-massive (hotter) component, $T_{\mathrm{h}}$, was set at $5800 \mathrm{~K}$. The indexes (h, c) refer to the hotter (less-massive) and cooler (moremassive) components respectively.

The values of the limb-darkening coefficients were derived from the stellar effective temperature and surface gravity, according to the given spectral type, by using the polynomial proposed by Díaz-Cordovés et al. (1995). During the process of optimisation, according to the temperature changes, we have an automatic recomputation of the limb-darkening. Following Lucy (1967), Rucinski (1969) and Rafert \& Twigg (1980), the gravity-darkening coefficients of the stars, $\beta_{\mathrm{h}, \mathrm{c}}$, and their albedos, $A_{\mathrm{h}, \mathrm{c}}$, were set at the values of 0.08 and 0.5 , respectively, appropriate for stars with convective envelopes.

The present analysis yields $F_{\mathrm{h}}>1$ for the filling coefficient in the critical Roche lobe, i.e., the overcontact configuration. Tidal effects are expected to contribute to synchronisation of the rotational and orbital periods. Therefore, in the inverse problem we adopted $f_{\mathrm{h}, \mathrm{c}}=\omega_{\mathrm{h}, \mathrm{c}} / \omega_{\mathrm{K}}=1.0$ for nonsynchronous rotation coefficients, where $f_{\mathrm{h}, \mathrm{c}}$ is the ratio of the angular rotation rate $\left(\omega_{\mathrm{h}, \mathrm{c}}\right)$ to the Keplerian $\left(\omega_{\mathrm{K}}\right)$ orbital revolution rate.

In previous versions of our programme, there were two different possibilities in the application of the model with respect to the treatment of the radiation law: the simple black-body theory, or the stellar atmosphere models by Carbon \& Gingerich (1969) (CG). Our current version of the programme for the light-curve analysis employs the new promising Basel Stellar Library (BaSeL). We have explored the "corrected" BaSeL model flux distributions, consistent with extant empirical calibrations (Lejeune et al. 1997, 1998), and with a large range of effective temperatures $2000 \mathrm{~K} \leq T_{\text {eff }} \leq 35000 \mathrm{~K}$, surface gravities, $3 \leq \log g \leq 5$ and metallicity, $-1 \leq[\mathrm{Fe} / \mathrm{H}] \leq 1$, where $[\mathrm{Fe} / \mathrm{H}]$ is the logarithmic metal abundance. The surface gravities can be derived very accurately from the masses and radii of close binary (CB) stars by solving the inverse problem of the light-curve analysis, but the temperature determination is related to the assumed metallicity and strongly depends on photometric calibration.

In the inverse problem, the fluxes are calculated in each iteration for the current values of temperatures and $\log g$ by interpolation in both of these quantities in the atmosphere tables, as an input, for a given metallicity of the $\mathrm{CB}$ components. The metallicity of the involved CB components can be different. It is because of this that we can use individual, different tables as an input for each star, and in this way choose the best calculations for its particular atmospheric parameters. Compared to Vaz et al. (1995), our two-dimensional flux interpolation in $T_{\text {eff }}$ and $\log g$ is based on the application of the bicubic spline interpolation (Press et al. 1992). This proved to be a good choice.

By choosing and fixing the particular input switch, the programme for the light-curve analysis can be simply redirected to the Planck or CG approximation, or to the more realistic BaSeL model atmospheres. Disagreement between individual $B$ and $V$ solutions decreases if we 
introduce the "corrected" BaSeL model flux distributions. A change in the assumed metallicity causes a noticable change in the predicted stellar effective temperature. The value of the chemical abundance of the components was obtained by checking several different values around solar metallicity. In the case of $\mathrm{U} P$ eg, the best fit of the $B$ and $V$ light curves was obtained with $[\mathrm{Fe} / \mathrm{H}]_{\mathrm{h}, \mathrm{c}}=0.2$ for the metallicity of the components. With this value, the individual $B$ and $V$ solutions are in good agreement. The results presented here are given within this stellar atmosphere approximation.

Among the light curves that were analysed, Zhai et al.'s (1984) photoelectric $B V$ light curves were the most symmetrical. For the estimation of the system's basic parameters we used the 1958 Binnendijk light curves (1960). In these light curves, the system is brighter in the maximum after a primary minimum than in the rest of the analysed light curves. This checking was done in a relative way, by measuring the light curve amplitude (max-min), and also by comparing the light levels in the light curve maxima. By that we have in mind that Binnendijk's observations were made with a non-standard filter combination, whose effective wavelengths were $\lambda_{\text {eff }}=5300 \AA$ ( $V$-filter) and $\lambda_{\text {eff }}=4420 \AA$ ( $B$-filter). The problem exists also for La Fara's (1952) observations, which were made with preJohnson filters, with effective wavelengths $\lambda_{\text {eff }}=5350 \AA$ $(V$-filter $)$ and $\lambda_{\text {eff }}=4500 \AA$ ( $B$-filter $)$. Other observations are in Johnson's system. The differences of effective wavelengths of La Fara's and Binnendijk's filters from Johnson's filters have as a consequence a wavelengthdependent influence on the light curve amplitude. This influence can be estimated and for both groups of observations lies in the interval $0^{\mathrm{m}} 01\left(\Delta V_{\mathrm{ampl}} \sim-0^{\mathrm{m}} 01\right.$; $\Delta B_{\mathrm{ampl}} \sim 0^{\mathrm{m}} 009-$ Binendijk's observations and $\Delta V_{\mathrm{ampl}} \sim$ -0. $008 ; \Delta B_{\text {ampl }} \sim 0$. 005 - La Fara's observations).

The obtained solutions show that Binnendijk's 1958 light level, at orbital phase 0.25 is very probably free of spot effects. In the analysis of these light curves, the inclination of the orbit was estimated to be $i \sim 76.1$, the filling coefficient for the critical Roche lobe $F_{\mathrm{h}} \sim 1.024$ and the temperature of the cooler (more massive) star $T_{\mathrm{c}} \sim 5600 \mathrm{~K}$.

Since the stars in the system have external convective envelopes, which can exhibit magnetic activity, we started the "spotted solution" by assuming that the components of U Peg have cool spots, of the same nature as solar magnetic spots. Moreover, since the results of the light-curve analysis depend on the choice of the adopted working hypothesis, the analysis was carried out within the framework of several hypotheses with spotted areas on the components.

We rejected those hypotheses which produced significantly different values of the parameters for the system and the active spotted areas, estimated by analysing the individual light curves in the $B$ and $V$ passbands. Finally we chose the Roche model with dark spotted areas on the more massive (cooler) component as the optimum solution. Within this hypothesis the analysis of the light curves yields mutually consistent parameters of the system and active region in the $B$ and $V$ passbands. In this case we obtained a very good fit of the observations for the whole set of analysed light curves without any changes in the system's basic parameters. Consequently, the presence of dark spotted areas on the cooler star can be taken as possible.

The system's basic parameters, obtained by analysing Binnendijk's (1960) light curves, were fixed in the inverseproblem solution for other, more or less deformed and asymmetrical light curves. In the subsequent analysis, these light curves are normalised to the reference light level of the 1958 Binnendijk light curves at orbital phase 0.25 , and their analysis was made with optimisation in the spot parameters.

The results indicate that the complex nature of the light-curve variations during the examined period can be explained by changes in the parameters of the spotted areas.

\section{Results}

The parameters derived from the light-curve analysis are listed in Table 1. The errors of the parameter estimates arise from the nonlinear least-squares method, on which the inverse-problem method is based. The first three rows of the table present the number of observations $n$, the final sum of squares of residuals between observed (LCO) and synthetic (LCC) light curves

$$
\sum_{i=1}^{n}\left(\mathrm{O}_{i}-\mathrm{C}_{i}\right)^{2}
$$

and the standard deviation of the observations

$\sigma=\sqrt{\frac{\sum_{i=1}^{n}\left(\mathrm{O}_{i}-\mathrm{C}_{i}\right)^{2}}{(n-1)}}$

In Table 1 , the indices $(B, V)$ denote the $B$ and $V$-filter observations, respectively.

In the same table the spot characteristics (spot temperature factor, $A_{\mathrm{S}}=T_{\mathrm{S}} / T_{*}$; longitude, $\lambda_{\mathrm{S}}$; latitude, $\varphi_{\mathrm{S}}$; angular radius, $\theta_{\mathrm{S}}$ ) are also given. Determination of these parameters is based on a simultaneous fitting of the available light curves in the $B$ and $V$ photometric bands for the different epochs of the observations with the same set of basic system parameters.

Finally, in Table 1 we present some important absolute parameters. They are derived from the mass-ratio of the components $\left(q=m_{\mathrm{c}} / m_{\mathrm{h}}=3.0186\right)$, orbital pe$\operatorname{riod}(P=0 \mathrm{~d} 37478048)$ and the semimajor orbital axis $\left(a_{\text {orb }}\left[R_{\odot}\right]=2.5325\right)$ estimated by Zhai et al. (1988) on the basis of the simultaneous solution of the light (Zhai et al. 1984) and radial velocity ( $\mathrm{Lu} 1985)$ curves.

Based on the Hipparcos parallax, Rucinski \& Duerbeck (1997) derived for the absolute visual magnitude of the system $M_{V}=3{ }^{\mathrm{m}} 69 \pm 0.42$. From our data in Table 1 , 
Table 1. Results of the analysis of the U Peg (1950, 1958, 1970, 1978, 1989) photometrical observations obtained by solving the light curve inversion problem for the Roche model with spotted areas on the more-massive (cooler) component

\begin{tabular}{|c|c|c|c|c|c|}
\hline Quantity & 1958 & 1950 & 1970 & 1978 & 1989 \\
\hline$n$ & $438_{B} \quad 447_{V}$ & $40_{B} \quad 40_{V}$ & $1417_{B} \quad 1421_{V}$ & $217_{B} \quad 215_{V}$ & $82_{V}$ \\
\hline$\Sigma(\mathrm{O}-\mathrm{C})^{2}$ & $0.0431_{B} \quad 0.0288_{V}$ & $0.0021_{B} \quad 0.0017_{V}$ & $0.1928_{B} \quad 0.1685_{V}$ & $0.0043_{B} \quad 0.0017_{V}$ & $0.0019_{V}$ \\
\hline$\sigma$ & $0.0099_{B} \quad 0.0080_{V}$ & $0.0074_{B} \quad 0.0067_{V}$ & $0.0117_{B} \quad 0.0109_{V}$ & $0.0045_{B} \quad 0.0067_{V}$ & $0.0048_{V}$ \\
\hline$A_{\mathrm{S}}^{1}=T_{\mathrm{S}}^{1} / T_{\mathrm{c}}$ & $0.84 \pm 0.01$ & $0.68 \pm 0.05$ & $0.776 \pm 0.005$ & $0.75 \pm 0.05$ & $0.73 \pm 0.03$ \\
\hline$\theta_{\mathrm{S}}^{1}$ & $11.2 \pm 0.2$ & $11.0 \pm 1.2$ & $29.9 \pm 0.2$ & $11.2 \pm 0.6$ & $30.8 \pm 0.4$ \\
\hline$\lambda_{\mathrm{S}}^{1}$ & $350.2 \pm 2.4$ & $25.5 \pm 5.1$ & $323.8 \pm 3.7$ & $227.4 \pm 2.9$ & $69.6 \pm 1.5$ \\
\hline$\varphi_{\mathrm{S}}^{1}$ & $24.5 \pm 7.4$ & $15.2 \pm 9.2$ & $83.6 \pm 0.3$ & $-14.2 \pm 4.6$ & $-47.7 \pm 0.6$ \\
\hline$A_{\mathrm{S}}^{2}=T_{\mathrm{S}}^{2} / T_{\mathrm{c}}$ & $0.73 \pm 0.06$ & $0.76 \pm 0.07$ & $0.76 \pm 0.06$ & $0.65 \pm 0.08$ & $0.73 \pm 0.04$ \\
\hline$\theta_{\mathrm{S}}^{2}$ & $12.6 \pm 0.5$ & $7.9 \pm 0.8$ & $9.3 \pm 1.0$ & $15.2 \pm 0.7$ & $9.0 \pm 0.4$ \\
\hline$\lambda_{\mathrm{S}}^{2}$ & $220.5 \pm 2.2$ & $202.4 \pm 6.4$ & $203.8 \pm 3.1$ & $44.5 \pm 2.3$ & $183.2 \pm 2.3$ \\
\hline$\varphi_{\mathrm{S}}^{2}$ & $23.6 \pm 5.6$ & $6.0 \pm 7.0$ & $16.0 \pm 2.1$ & $67.0 \pm 1.6$ & $18.2 \pm 1.7$ \\
\hline$T_{\mathrm{c}}$ & $5600 \pm 8$ & & & & \\
\hline$F_{\mathrm{h}}$ & $1.024 \pm 0.001$ & & & & \\
\hline$i$ & $76.1 \pm 0.1$ & & & & \\
\hline$u_{\mathrm{h}}$ & $0.74_{B} \quad 0.66_{V}$ & & & & \\
\hline$u_{\mathrm{c}}$ & $0.75_{B} \quad 0.67_{V}$ & & & & \\
\hline$\Omega_{\mathrm{h}, \mathrm{c}}$ & 6.5489 & & & & \\
\hline$\Omega_{\text {in }}$ & 6.6409 & & & & \\
\hline$\Omega_{\text {out }}$ & 6.0217 & & & & \\
\hline$f_{\text {over }}[\%]$ & 14.87 & & & & \\
\hline$R_{\mathrm{h}}$ & 0.275 & & & & \\
\hline$R_{\mathrm{c}}$ & 0.454 & & & & \\
\hline$L_{\mathrm{h}} /\left(L_{\mathrm{h}}+L_{\mathrm{c}}\right)$ & $0.325_{B} \quad 0.310_{V}$ & $0.323_{B} \quad 0.310_{V}$ & $0.335_{B} \quad 0.320_{V}$ & $0.327_{B} \quad 0.313_{V}$ & $0.322_{V}$ \\
\hline$a_{\text {orb }}\left[R_{\odot}\right]$ & 2.5325 & & & & \\
\hline $\mathcal{M}_{\mathrm{h}}\left[M_{\odot}\right]$ & 0.39 & & & & \\
\hline $\mathcal{M}_{\mathrm{c}}\left[M_{\odot}\right]$ & 1.17 & & & & \\
\hline $\mathcal{R}_{\mathrm{h}}\left[R_{\odot}\right]$ & 0.75 & & & & \\
\hline $\mathcal{R}_{\mathrm{c}}\left[R_{\odot}\right]$ & 1.23 & & & & \\
\hline $\log g_{\mathrm{h}}$ & 4.27 & & & & \\
\hline $\log g_{\mathrm{c}}$ & 4.32 & & & & \\
\hline$M_{b o l}^{\mathrm{h}}$ & 5.39 & & & & \\
\hline$M_{b o l}^{\mathrm{c}}$ & 4.46 & & & & \\
\hline
\end{tabular}

\section{FIXED PARAMETERS:}

$T_{\mathrm{h}}=5800 \mathrm{~K}$ - temperature of the less-massive (hotter) component.

$T_{\mathrm{c}}=5600 \mathrm{~K}$ - temperature of the more-massive (cooler) star.

$f_{\mathrm{h}}=f_{\mathrm{c}}=1.00-$ nonsynchronous rotation coefficients of the components.

$F_{\mathrm{h}}=1.024-$ filling coefficient for the critical Roche lobe of the hotter star.

$i=76.1$ - orbit inclination (in arc degrees).

$q=m_{\mathrm{c}} / m_{\mathrm{h}}=3.019-$ mass-ratio of the components.

$\beta_{\mathrm{h}, \mathrm{c}}=0.08-$ gravity-darkening coefficients of the components.

$A_{\mathrm{h}, \mathrm{c}}=0.5$ - albedo coefficients of the components.

$[\mathrm{Fe} / \mathrm{H}]_{\mathrm{h}, \mathrm{c}}=0.2-$ accepted metallicity of the components.

Note: $n$ - number of observations, $\Sigma(\mathrm{O}-\mathrm{C})^{2}-$ final sum of squares of residuals between observed (LCO) and synthetic (LCC) light curves, $\sigma$-standard deviation of the observation, $A_{\mathrm{S}}^{1,2}-$ spots' temperature coefficients, $\theta_{\mathrm{S}}^{1,2}, \lambda_{\mathrm{S}}^{1,2}$ and $\varphi_{\mathrm{S}}^{1,2}-$ spots' angular dimensions, longitudes and latitudes (in arc degrees), $u_{\mathrm{h}, \mathrm{c}}-$ limb-darkening coefficients of the components, $\Omega_{\mathrm{h}, \mathrm{c}}-$ dimensionless surface potentials of the common photosphere, $\Omega_{\text {in }}, \Omega_{\text {out }}$ - the potentials of the inner and outer contact surfaces respectively, $f_{\text {over }}[\%]=100 \cdot\left(\Omega_{1,2}-\Omega_{\text {in }}\right) /\left(\Omega_{\text {out }}-\Omega_{\text {in }}\right)$ - degree of overcontact, $R_{\mathrm{h}, \mathrm{c}}$ - polar radii of the components in units of the distance between the component centres, $L_{\mathrm{h}} /\left(L_{\mathrm{h}}+L_{\mathrm{c}}\right)$ - luminosity of the hotter star (including spots on the cooler one), $a_{\mathrm{orb}}\left[R_{\odot}\right]-$ orbital semi-major axis in units of the solar radius, $\mathcal{M}_{\mathrm{h}, \mathrm{c}}\left[M_{\odot}\right]-$ masses of components in solar units, $\mathcal{R}_{1,2}\left[R_{\odot}\right]-$ mean radii of stars in solar units, $\log g_{\mathrm{h}, \mathrm{c}}-$ logarithm (base 10) of the mean surface acceleration (effective gravity) for components and $M_{\mathrm{bol}}^{\mathrm{h}, \mathrm{c}}$ - absolute bolometric magnitudes of stars. 
including the bolometric corrections, we derived for the absolute visual magnitude of the system $M_{V} \sim 4^{\mathrm{m}} 13$. In the limits of the error this value agrees with the absolute visual magnitude of the system based on the Hipparcos parallax.

The solution for each individual light curve is presented in Fig. 1, where the optimum fit of the observed light curves (LCO) to the synthetic ones (LCC) is shown. The reference light level of Binnendijk's 1958 light curve at orbital phase 0.25 , for the unspotted configuration of the system is denoted by a dashed line. The rest of the light curves are scaled to this light level. The $\mathrm{O}-\mathrm{C}$ residuals between the observed (LCO) and optimum synthetic (LCC) light curves are given on the left-hand sides of Fig. 2. The right-hand side on these panels shows the view of Roche models of U Peg obtained with the parameters estimated by analysing the corresponding light curves. Using such plots, one sees how a CB system would look at a certain orbital phase, chosen so that the spots are visible.

It is evident from Table 1 and from Figs. 1 and 2 (left) that the Roche model with spot areas on the cooler component gives a satisfying fit of the analysed light curves.

In the case of two spotted areas on the cooler component, the Roche model with the system's basic parameters that are constant for the whole set of the analysed light curves satisfyingly fits the observations. This indicates that the complex nature of the light-curve variations during the examined period can be explained by the evolution and motion of spotted areas across the stellar surfaces.

The solutions presented here show that $\mathrm{U}$ Peg is in an overcontact configuration $\left(f_{\text {over }}[\%] \sim 14.9 \%\right)$, with a small temperature difference between the components $\left(\Delta T=T_{\mathrm{h}}-T_{\mathrm{c}} \sim 200 \mathrm{~K}\right)$ proving a good thermal contact between the components. The mass-ratio estimated to $q=m_{\mathrm{c}} / m_{\mathrm{h}} \sim 3.019$ suggests a significant energy transfer between the components through the connecting neck of the common envelope.

The size of a spot area can be used as an indicator of the system's activity. The analysis of the symmetric light curves from 1950 shows only the presence of small spotted areas (located relatively close to the stellar equator) which can produce a very small light-curve asymmetry. It appears that the system is very probably in the minimum of the spot activity during that period. Later, the activity of the system increases, and the analysis of the light curves from 1958 gives large spots on the same stellar hemispheres which cover a significant part of the stellar surfaces. So, their presence leads to a conspicuous asymmetry in the light curves. The spot locations from 1970 produce a light-curve asymmetry and a change in the light level at the maxima. The larger spot is located at high latitude in the polar region, and the small one is near the stellar equator. It seems that the system's activity decreases after that year. The spot locations from 1978 practically do not affect the symmetric shape of the light curves, but they change the light level at the maxima. The observations made during November 1989 at the
Observatorio Astronómico Nacional at San Pedro Mártir with 84-cm reflecting telescope in Johnson's $V$ filter show large activity in the system again. It is possible that large spot areas at high latitudes (near stellar polar regions) correspond to an enhanced activity of the system.

Finally, Fig. 3 presents observations of U Peg drawn together for the $B$ and $V$ filters respectively, and the corresponding colour-index $B-V$ drawn for each epoch separately. During the analysed period, the main variations in the light curves are in the height of both maxima as well as in the depth of the light-curve minima. The $B-V$ colourindex for the whole set of the analysed light curves shows some reddening around the light-curve minima. This effect may be attributed to the temperature differences between the components, and partially to the influence of spot active regions.

\section{Discussion and conclusions}

Both presentations of our results obtained in the analysis of U Peg light curves between 1950 and 1989 (Table 1 and the corresponding figures) show that the Roche model with spot areas on the cooler component provides a satisfying fit of the observations. During that period, the main variations in the light curves can be explained by the change in the temperature contrast, and the position and the size of spot areas on the cooler component. Having in mind that we used the same basic system parameters for different curves, these results indicate that the complex nature of the light-curve variations may be attributed to variable starspot activity.

Moreover, several attempts were made to fit the observations by assuming a spot model with spots on both components. In that case we can obtain fits which are similar in quality, but for this we need some variations of the system's basic parameters derived by analysing the individual light curves around a mean value. We consider that case as less reliable, such that we feel it to be possibly excluded.

The present analysis shows that the changes in the light curves can be relatively well interpreted by the development and migration of spot regions on the cooler component. The model can successfully simulate the observed light curves which cover an interval of 39 years.

Our analysis shows that during the deeper (primary) minimum the cooler (more massive and larger) component eclipses the hotter (less massive and smaller) one. The solutions presented here show that $\mathrm{U} P$ eg is in the over-contact configuration $\left(f_{\text {over }}[\%] \sim 14.9 \%\right.$ ), with a small temperature difference between the components ( $\Delta T=T_{\mathrm{h}}-T_{\mathrm{c}} \sim 200 \mathrm{~K}$ ) indicating a good thermal contact between the components. The mass-ratio estimated to $q=m_{\mathrm{c}} / m_{\mathrm{h}} \sim 3.019$ suggests a significant energy transfer between the components. 

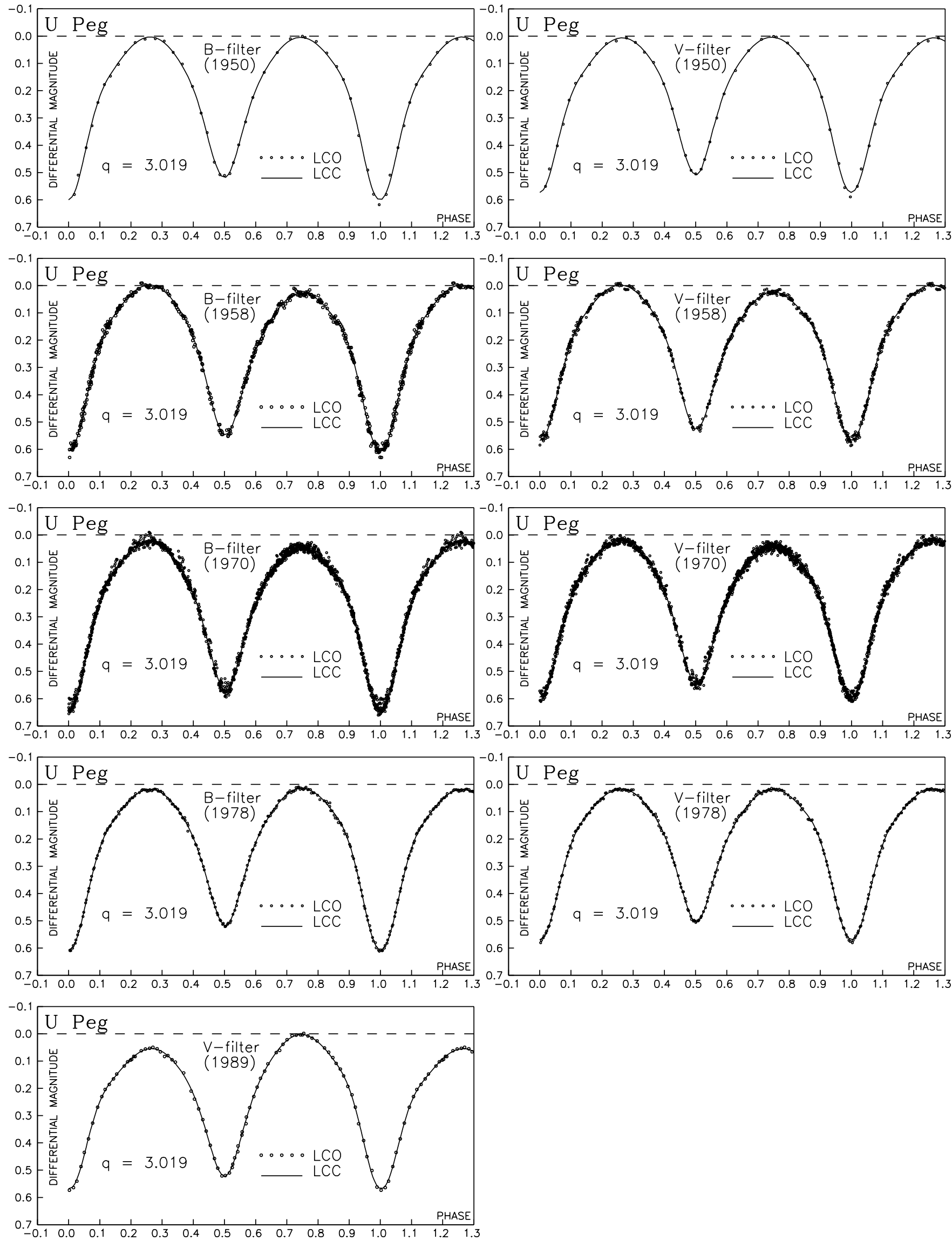

Fig. 1. Observed (LCO) and final synthetic (LCC) light curve of U Peg. The reference light level of Binnendijk's 1958 light curve, at orbital phase 0.25 , for the unspotted configuration of the system is shown by the dashed line 

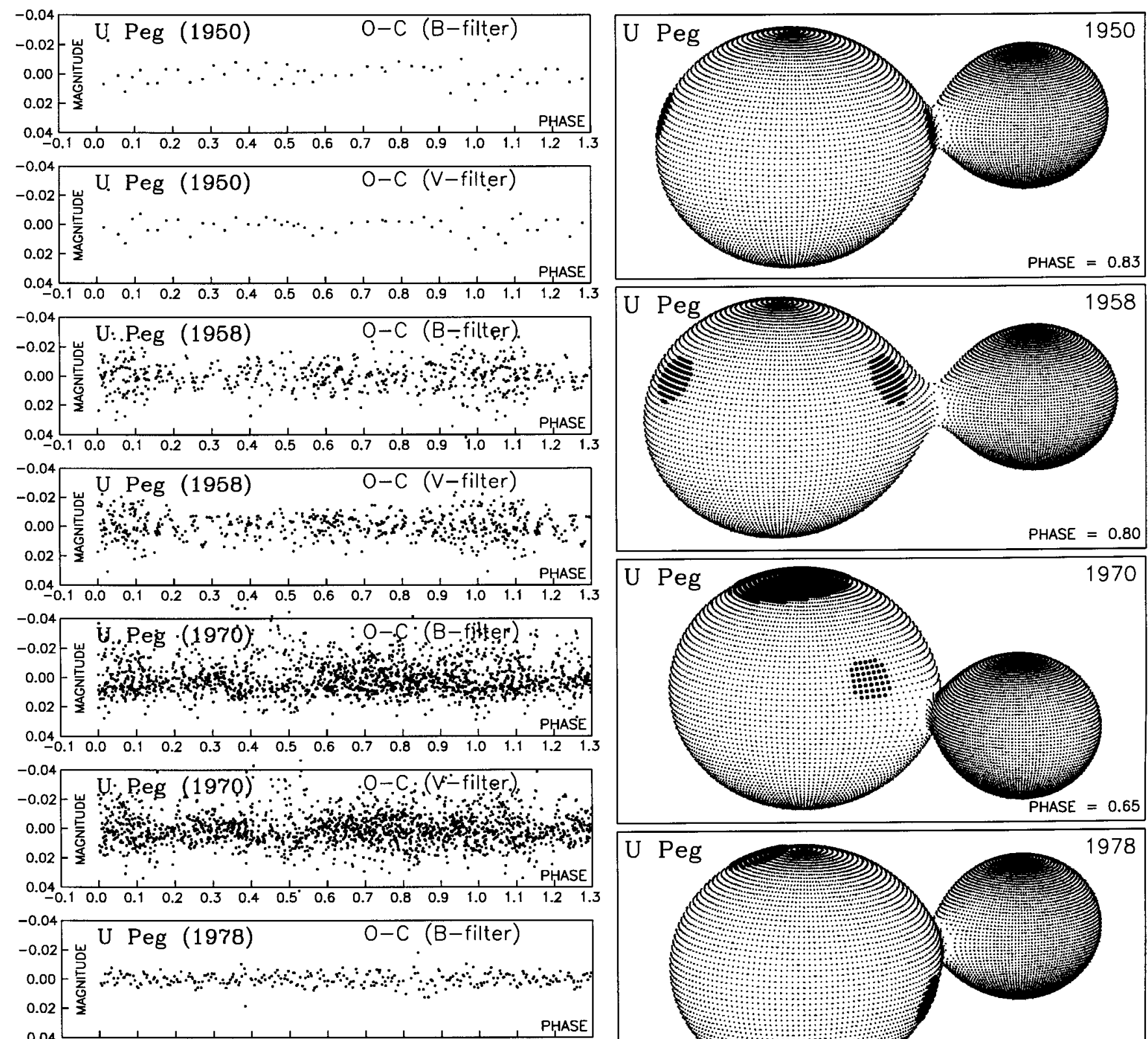

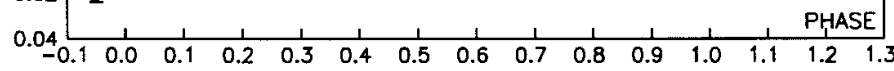

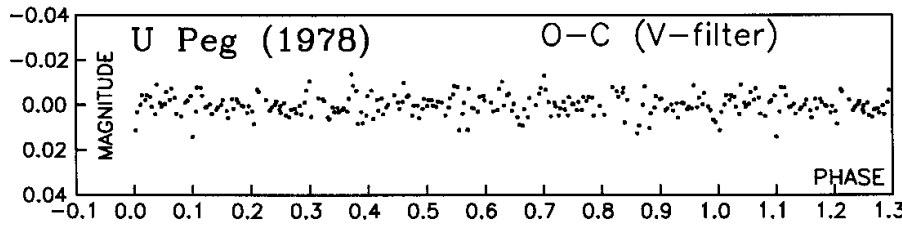
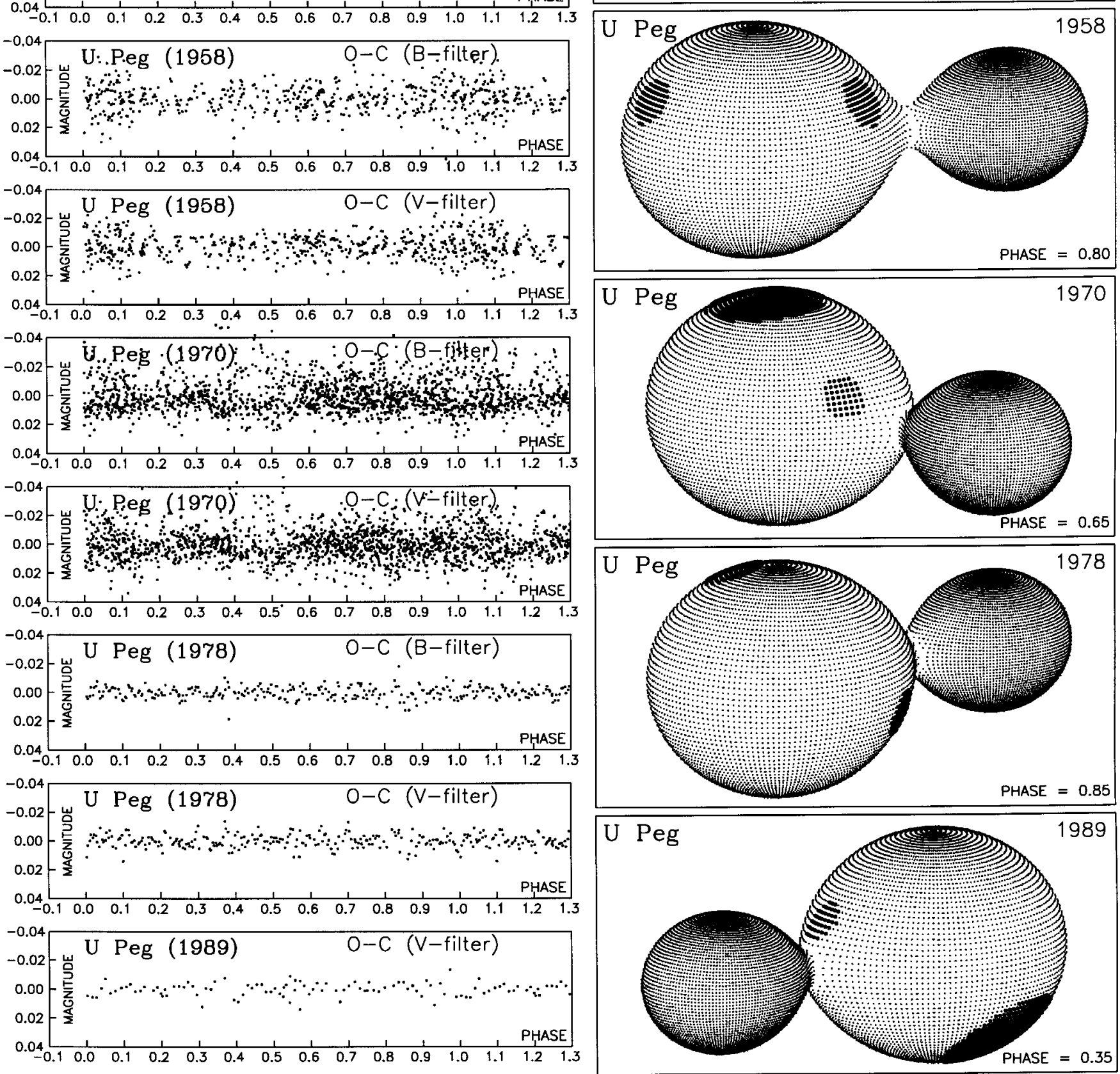

Fig. 2. Left: Final O-C residuals obtained by solving the inverse problem within the framework of the Roche model with spotted areas on the cooler component; Right: The view of the Roche model for U Peg at the noted orbital phase, obtained with parameters estimated by solving the inverse problem 

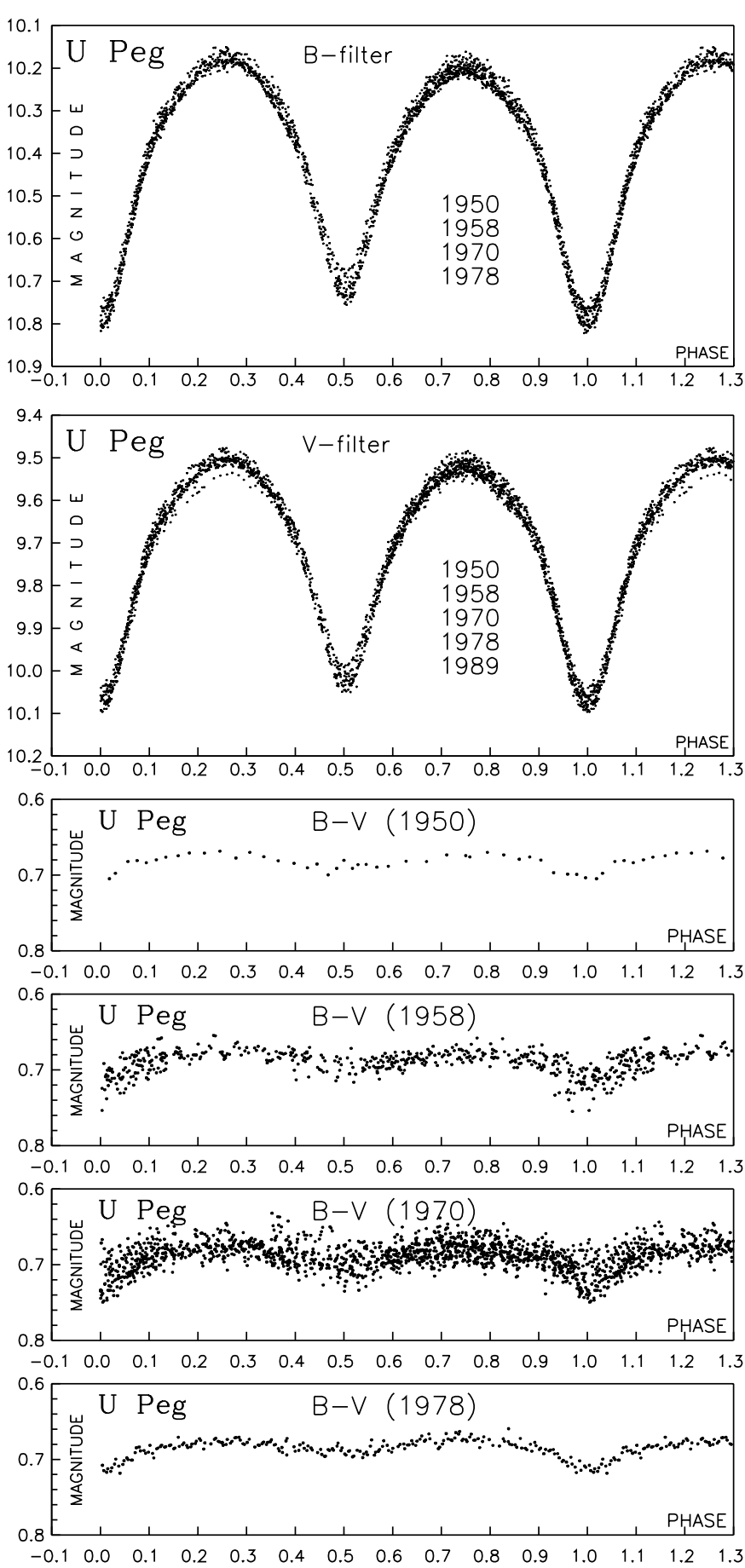

Fig. 3. Observations of U Peg drawn together for $B$ and $V$ filters respectively, and corresponding $B-V$ colour-index drawn for each year separately

Having in mind the large changes in the shape of the light curves, future photometric observations of this interesting system are of great interest.
Acknowledgements. Three of us (G. D., S. E. \& N. M.) have been partially supported by the Ministry for Sciences and Technology of Serbia through the project "Astrometrical, Astrodynamical and Astrophysical investigations". H. R.-L. \& P. R. acknowledge some financial support from Athens University and the National Observatory of Athens.

\section{References}

Adams, W. S., Joe, A. H., \& Sanford, R. F. 1924, PASP, 36, 139

Adams, W. S., Joe, A. H., Humason, M. L., \& Brayton, A. M. 1924, ApJ, 81, 187

Binnendijk, L. 1960, AJ, 65, 88

Carbon, D., \& Gingerich, O. 1969, in Theory and Observation of Normal Stellar Atmospheres, ed. O. Gingerich (MIT Press, Cambridge), 377

Chandler, S. C. 1895, AJ, 15, 181

Díaz-Cordovés, J., Claret, A., \& Giménez, A. 1995, A\&AS, 110,329

Djurašević, G. 1992a, Ap\&SS, 196, 241

Djurašević, G. 1992b, Ap\&SS, 197, 17

Djurašević, G., Zakirov, M., Hojaev, A., \& Arzumanyants, G. 1998, A\&AS, 131, 17

La Fara, R. 1952, ApJ, 115, 14

Lafta, S. J., \& Grainger, J. F. 1986, Ap\&SS, 121, 61

Lejeune, T., Cuisinier, F., \& Buser, R. 1997, A\&AS, 125, 229

Lejeune, T., Cuisinier, F., \& Buser, R. 1998, A\&AS, 130, 65

Lu, W. X. 1985, PASP, 97, 1086

Lucy, L. B. 1967, Zs. f. Ap., 65, 89

Lucy, L. B., \& Wilson, R. E. 1979, ApJ, 231, 502

Marquardt, D. W. 1963, J. Soc. Ind. Appl. Math., 11, No. 2, 431

Maupomé, L., Rodríguez, E. Hobart, M. A., Peña, J. H., \& Peniche, R. 1991, Rev. Mexicana Astron Astrof., 22, 235

Press, W. H., Teukolsky, S. A., Vetterling, W. T., \& Flannery, B. P. 1992, Numerical Recipes in Fortran, The Art of Scientific Computing, Second Edition (Cambridge University Press, New York), 120

Rafert, J. B., \& Twigg, L. W. 1980, MNRAS, 139, 78

Rigterink, P. V. 1972, AJ, 77, 319

Rucinski, S. M. 1969, Acta Astr., 19, 245

Rucinski, S. M., \& Duerbeck, H. W. 1997, PASP, 109, 1340

Russo, G., Sollazzo, C, Maceroni, C., \& Milano, L. 1982, A\&AS, 47, 211

Struve, O., Horak, H. G., Canavaggia, R., Kourganoff, V., \& Colachevich, A. 1950, ApJ, 111, 658

Vaz, L. P. R., Anderson, J., \& Rabello Soares, M. C. A. 1995, A\&A, 301, 69

Wilson, R. E., \& Devinney, E. J. 1971, ApJ, 166, 605

Zhai, D. S., Leung, K. C., \& Zhang, R. X. 1984, A\&AS, 57, 487

Zhai, D. S., Lu, W. X., \& Zhang, X. Y. 1988, Ap\&SS, 146, 1 